\title{
Hands-On Learning of Wireless Communication Principles Using Software- Defined Radio Experiments and LabVIEW
}

\section{Dr. Ismail Guvenc, Florida International University}

Dr. Ismail Guvenc (senior member, IEEE) received his Ph.D. degree in electrical engineering from University of South Florida in 2006, with an outstanding dissertation award. He was with Mitsubishi Electric Research Labs during 2005, and with DOCOMO Innovations Inc. between 2006-2012, working as a research engineer. Since August 2012, he has been an assistant professor with Florida International University. His recent research interests include heterogeneous wireless networks and future radio access beyond $4 \mathrm{G}$ wireless systems. He has published more than 100 conference/journal papers and book chapters, and several standardization contributions. He co-authored/co-edited three books for Cambridge University Press, served as an editor for IEEE Communications Letters (2010-2015) and IEEE Wireless Communications Letters (2011-present), and as a guest editor for several other journals. Dr. Guvenc is an inventor/coinventor in 23 U.S. patents, and has another 4 pending U.S. patent applications. He is a recipient of the 2014 Ralph E. Powe Junior Faculty Enhancement Award and 2015 NSF CAREER Award.

\section{Dr. Natalie Paul, Florida International University}

Dr. Natalie Paul has a degree in Curriculum and Instruction with a specialization in Learning Technologies. She, also, has a background in Mathematics Education and Engineering.

\section{Mr. Rhys Bowley, National Instruments}

Rhys is an engineer working at National Instruments in a role that focuses on wireless communications in education. Rhys graduated with a Masters (MEng Hons) in Communications and Electronic Engineering from Cardiff University, where he spent a short time working as a researcher in loadpull characterisation techniques for mobile device power amplifiers. During his time at Cardiff he also taught lab classes in LabVIEW programming for both under graduate and post graduates alike. His passion is that wireless communications in education should not get left in the past, and that the most effective way to fuel world changing discoveries is to have research and industry ready undergraduates with real world experience.

\section{Dr. Maria Lorelei Fernandez, Florida International University}

Maria L. Fernandez has a PhD in Mathematics Education and is currently engaged in research to better understand engineering students' learning from and perceptions of experiences involving the use of novel hands-on experimentation in wireless communications. Her research has focused on experiences promoting undergraduate student learning and development, particularly with preservice mathematics teachers. Also, she has conducted work on the use of technology for student learning of mathematics. 


\section{Hands-on Learning of Wireless Communication Principles Using Software Defined Radio Experiments and LabVIEW}

With the rapid proliferation of millions of smartphones, the adoption of the latest 4G LTE technology worldwide, and the emergence $5 \mathrm{G}$ broadband wireless technologies, wireless communications have become an integral part of every person's daily life and will continue to be as such in the foreseeable future. Due to this remarkable surge in wireless technologies, a strong need for developing a flexible, hands-on laboratory platform to teach a wide variety of wireless techniques has emerged. Indeed, current educational systems worldwide and in the United States teach wireless communications by mostly (and sometimes exclusively) focusing on the theory. This approach is perceived as dry by the students who often find it hard to connect the rather complex theoretical abstractions to their everyday "fun" interaction with smartphones. Recently, software-defined radio $(\mathrm{SDR})^{1}$ and cognitive radio $(\mathrm{CR})^{2}$ concepts that allow the developing of programmable and affordable wireless systems have gained significant interest in the wireless community. The introduction of new highly reconfigurable and affordable SDR-based hardware modules, such as the popular Universal Software Radio Peripheral (USRP) platform, is seen as a promising approach to building a hands-on wireless laboratory.

To this end, faculty at the University of Texas at Austin and National Instruments (NI) have recently introduced a series of lab experiments for teaching wireless communication principles using the USRP SDR platforms ${ }^{3,4}$. First, fundamental theory of wireless communications such as digital modulation formats, synchronization, and equalization are introduced in the class. Later, students implement these theoretical concepts in the lab using the National Instrument's USRP SDR platforms. At Florida International University, this same curriculum was used to teach wireless communications to undergraduate and graduate students. The goal of this paper will be to summarize the experiences and lessons learned from teaching this course during the years 2014 and 2015 to approximately 15 undergraduate and 20 graduate students. To collect data, pre-class and post-class surveys were conducted with the students at the beginning and end of each semester. Moreover, post-lab online surveys were made to collect data explicitly for the hands-on lab experiments. Frequently, students have commented on the lack of background for using LabVIEW Communications software. For example, one student indicated that "Since LabVIEW is new to many of us, it would be greatly helpful if the lab process were taken step-by-step". Some other students suggested that the lab manual should include more detailed instructions. In general, survey results highlighted the importance of using hands-on components in the course: "USRP Lab experiments greatly help by showing visually how the concepts we learn in class are applicable" and "[USRP labs were] extremely useful, they were a big help in grasping the concepts".

The rest of this paper is organized as follows. In the following section, we provide an overview of the existing literature on hands-on education for wireless communications. Next, motivations for using hands-on education to teach wireless communication concepts, in particular using SDR platforms, is presented. Then, an overview of how the USRP course is taught at the Florida International University is described. After, an introduction to the data that 
has been collected while teaching the course at the same university and the lessons learned are detailed. Finally, the last section provides concluding remarks.

\section{Literature Review}

Wireless communications has become an attractive field for engineering students ${ }^{5}$. Working with wireless systems can be highly motivational because of the increase in the number of students familiar with wireless devices and wireless networks ${ }^{6}$. In addition, the growth of the cellular and mobile job markets further promotes enrollments in wireless communication courses $^{5}$. Thus, there is a need for wireless communications courses to provide experiences comparable to the future work environment for students. This educational objective of wireless communications courses can be met through the implementation of hands-on learning, which includes lab experiments. Researchers suggest that effective courses provide hands-on learning experiences ${ }^{7}$. Students take a professional approach to wireless network design through simulations $^{8}$. Moreover, successful courses encourage student responsibility and ownership for projects 9 .

Instruction in theory coupled with real-life applications is the overarching goal of any engineering course. The translation of the abstract theory into real-life problems is critical in engineering education ${ }^{10}$. Generally, in wireless communications courses students only learn theoretical background knowledge because they do not have access to the equipment and technologies that accompany $\mathrm{it}^{11}$. However, incorporating hands-on experiments in these courses can increase student learning ${ }^{12}$. Additionally, hands-on learning in a wireless communications course can motivate students. Researchers have found enthusiasm and interest from students who participated in lab experiments in wireless communications courses ${ }^{12}$. The abstract theory can be effectively conveyed through experiments ${ }^{10}$.

Furthermore, hands-on activities work well with a project-based curricula, which has been successful in research studies ${ }^{5}$. Guzelgoz and Arslan ${ }^{12}$ surveyed students before the final exam in a wireless communications lab course. Their results demonstrated how students can learn the difficult theoretical knowledge with the help of labs. Also, students agreed that the lab component made the course more interesting. Moreover, Dawy, Husseini, Yaacoub, and Al$\mathrm{Kanj}^{11}$ evaluated a wireless communications course with an end-of-term assessment about the 10 lab experiments conducted by students throughout the course. The researchers used an anonymous online survey to gauge learning outcomes of 15 students per term. Results indicated that all outcomes were achieved with a high level of satisfaction from students. Thus, there is a need for more lab experiments to parallel content in wireless communications courses. Students want more hands-on work that can link theoretical knowledge to real-life applications ${ }^{10}$.

\section{Hands-on Wireless Communications Education with SDR and LabVIEW}

\section{Focus on Discovery}

Academia's primary focus is, at a high level, the discovery of the unknown. Often research efforts and teaching efforts are discussed separately, as though they are opposing topics. However, they are fundamentally the same pursuit with different scope; researchers work to discover what nobody knows whereas students work to discover what they do not know. What is important in both scenarios is the rate of discovery. This is often apparent in teaching as educators strive to cover all material within a 15 week semester. 
Discovery requires experimentation and insight. Students and researchers alike must be able to quickly and easily iterate on ideas, test theories, and instantly see the results. Within the context of this paper LabVIEW Communications is the platform for discovery - a graphical system design software.

\section{"I hear and I forget. I see and I remember. I do and I understand."}

Many educators understand the benefits of adding a hands-on practical element to their classes. Students who are able to explore and experiment with theory will retain more information and develop intuitive analytical skills. Theory is critically important, but a balance must be struck with students in order to maintain engagement and interest. With a goal of producing industry and research ready graduates in the field of wireless communications, we must remember that early successes in a specific field can have a significant impact on students' choices of career.

\section{Specific problems in Wireless Communications}

Wireless communications suffers two compounding problems. Firstly, the theory of high frequency electromagnetic signals is complex. High frequency phenomena often defy the macroscale logic students see in the world around them on a daily basis. Furthermore, wireless communications systems suffer from numerous influences caused by the transmission and reception hardware itself, as well as the infinitely variable parameters of the channel and environment. These real-world caveats mean that simulation alone is ineffective as the only mechanism for students to gain experience in designing viable communications systems.

The second part of the problem is cost. Radio and microwave frequency instrumentation can easily run into the hundreds of thousands of dollars. These large instruments require great care so are not robust enough for the teaching laboratory. Furthermore more, they often require expertise in multiple low level programming languages so they are not accessible to undergraduate students because the learning curve is too steep for semester based courses.

\section{The NI Solution for Teaching Wireless Communications}

Graphical system design is accelerating discovery in many areas of teaching, research and industry. NI creates turnkey teaching solutions using industry standard tools. These teaching solutions are comprised of LabVIEW graphical system design software, tightly integrated NI USRP hardware and ready to run course material ${ }^{14}$. LabVIEW Communications System Design Suite unifies the design approach from algorithm to real world prototype. NI USRP is a family of broad band software defined radios which are programmed by the user. NI works with professors who have a long history of teaching wireless communications to develop course material and laboratory manuals that accompany the hardware and software. The lab course described in the next section has been created using one of these teaching solutions from NI.

\section{Wireless Digital Communications Course}

The data to be presented is collected during the Wireless Digital Communications course taught at the Department of Electrical and Computer Engineering at a Florida International 
University. The course is taught over two semesters, during Spring 2014 (9 graduate and 13 undergraduate students) and Fall 2015 (12 graduate and 4 undergraduate students). During both semesters, pre-class and post-class surveys were implemented with the students to collect information about the course. Moreover, during the Fall 2015 semester, survey data was collected from the students following each of the lab experiments. The lab benches for the experiments as well as the USRP equipment from NI are illustrated in Figure 1.

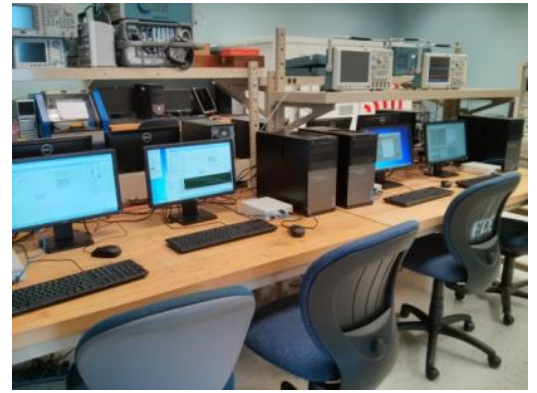

(a) Lab benches with USRPs.

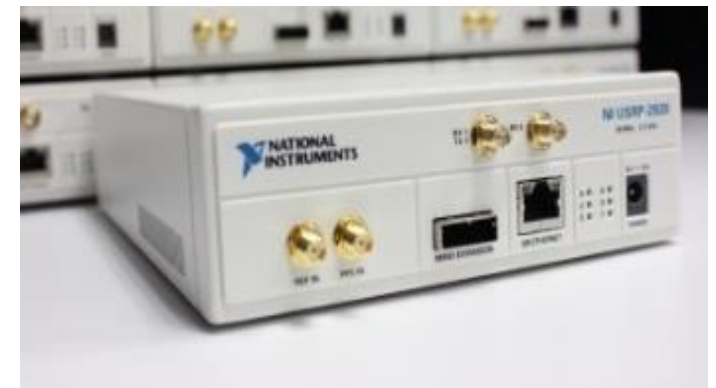

(b) USRP from National Instruments.

Figure 1 Lab benches for experiments with USRPs, and a closer view of USRP SDR from National Instruments.

The course involves two components: In-class lecturing (two 75 minute sessions per week), and bi-weekly lab experiments. The students are grouped in pairs. There are a total of 5 lab experiments that the students need to finish. Each lab experiment has a pre-lab component which the students have to finish before coming to the lab. This approach maximizes the student's time in the lab for exploration and experimentation. After a three-hour lab experiment, students also have to prepare a post-lab report. The lab sessions are arranged so that they do not happen before covering the related theory in the class, and that students are well prepared with the required knowledge prior to the experiments. The students are also expected to form groups and work on a hands-on course project, and the lab experiments help the students to gain relevant experience before starting their course projects. The combination of lecture and lab experiments where students work together promoted hands-on and collaborative learning. Distribution of the labs and project presentations throughout the semester are summarized in Table 1.

\section{Survey Results and Lessons Learned}

In this section, we will present and interpret the data collected from the pre/post class surveys and labs. Due to the internal review board (IRB) approval being available only for the 2015 offering of the course, most data presented in this paper will be limited to the data collected during the course that was offered in 2015. Overall, there were 16 students who filled out the pre-course survey, and 9 students who filled out the post-course survey. Prior to taking the course, most undergraduate students have taken Introduction to Communication Systems, and many of the graduate students have taken more advanced wireless communication courses at the graduate level. 
Table 1 Timeline for lab experiments and project presentations in a 15-week semester.

\begin{tabular}{|l|l|l|}
\hline Demo & $\begin{array}{l}\text { Implementation of FM radio and digital } \\
\text { transmitter/receiver (not graded) }\end{array}$ & Week 3 (all) \\
\hline Lab-1 & $\begin{array}{l}\text { Introduction to NI LabVIEW Communications } \\
\text { and NI USRP hardware }\end{array}$ & Week 4 (grad), Week 5 (undergrad) \\
\hline Lab-2 & $\begin{array}{l}\text { Modulation and detection, pulse shaping, and } \\
\text { matched filtering }\end{array}$ & Week 6 (grad), Week 7 (undergrad) \\
\hline Lab-3 & Synchronization & Week 8 (grad), Week 9 (undergrad) \\
\hline Lab-4 & Channel estimation and equalization & Week 10 (grad), Week 11 (undergrad) \\
\hline Lab-5 & Frame detection and frequency offset correction & Week 12 (grad), Week 13 (undergrad) \\
\hline Projects & Students demonstrate their course projects. & Weeks 14-15 \\
\hline
\end{tabular}

\section{Improvement in Learning Perceptions of the Students}

We identified 40 technical concepts that are of relevance to technical concepts taught throughout the course. The students are asked to indicate, on a scale of 1 to 5, how knowledgeable they are in each concept. This survey is conducted during the first week of the semester, and then again during the last week of the semester. Analysis of the data from these surveys is given in Table 2, where AVG refers to average of student's scores for each technical concept, STD refers to standard deviation, and Pre/Post refer to the data collected prior to the course and after the course, respectively. Based on the averaged student scores, the last column also shows how much the students perceptions have improved, at a percentage level over a scale of 5 , in each technical concept.

The data in Table 2 has many interesting insights related to how hands-on education sessions improve the learning experience of the students. For example, students' perceptions of their technical competence in LabVIEW Communications (25\%), USRP devices (33.8\%), and software defined radios (40\%) improved, which illustrates that hands-on labs can impact student learning in a positive manner. As a benchmark, it is worthwhile to see that gain for handover in wireless networks is only $2 \%$. Indeed, while this concept was studied during the first offering of the course in 2014, it was not taught during the second offering of the course in 2015. Therefore, there was close to no difference in the perceptions of the students at the end of the semester for this concept.

The hands-on lab experiments allowed students to connect technical concepts discussed in lecture to the usage of actual equipment. Remarkably, some of the technical concepts that are at the core of the lab experiments had considerably larger gains compared to some other concepts. Students may have had these larger gains in some areas as opposed to others because of how they translated these concepts in their lab work. For example, synchronization is the 
Table 2 Statistical data related to students' own perception of how they think they know different concepts in wireless communications, before and after the course.

\begin{tabular}{|c|c|c|c|c|c|}
\hline & AVG (Pre) & STD (Pre) & AVG (Post) & STD (Post) & Gain (\%) \\
\hline LabVIEW Communications & 1.8 & 0.9 & 3 & 0.72 & 25.0 \\
\hline Matlab & 3.1 & 0.8 & 3.9 & 1.02 & 15.5 \\
\hline NI USRP devices & 1.3 & 0.6 & 3 & 0.76 & 33.8 \\
\hline Software defined radio & 1.7 & 1.0 & 3.7 & 1.12 & 40.3 \\
\hline BPSK modulation & 2.6 & 1.1 & 4 & 1.08 & 27.5 \\
\hline QPSK modulation & 2.6 & 1.1 & 4 & 1.08 & 27.5 \\
\hline Bit error rate calculation & 2.4 & 1.3 & 4 & 1.38 & 31.3 \\
\hline Pulse shaping & 1.9 & 0.9 & 3.1 & 0.72 & 24.5 \\
\hline Channel impulse response & 2.1 & 1.2 & 3.1 & 0.76 & 19.5 \\
\hline Wireless propagation channel & 2.4 & 1.0 & 3.4 & 0.88 & 20.5 \\
\hline Time selectivity & 2.1 & 1.1 & 3.3 & 0.77 & 23.5 \\
\hline Frequency selectivity & 2.1 & 1.2 & 3.6 & 0.76 & 30.8 \\
\hline Doppler spread & 2.3 & 1.1 & 3.4 & 1.19 & 21.8 \\
\hline Wireless channel estimation & 1.8 & 1.0 & 4 & 0.87 & 43.8 \\
\hline Wireless synchronization & 1.8 & 0.9 & 3.9 & 0.87 & 43.0 \\
\hline Wireless equalization & 1.8 & 1.0 & 3.5 & 1.06 & 33.8 \\
\hline OFDM modulation & 1.9 & 0.9 & 3.3 & 0.88 & 28.5 \\
\hline Cyclic prefix & 1.6 & 1.0 & 3.7 & 1.06 & 41.5 \\
\hline Frequency domain equalization & 1.8 & 0.8 & 3.5 & 0.73 & 35.0 \\
\hline MIMO systems & 2.3 & 1.2 & 3.1 & 1.03 & 17.0 \\
\hline Alamouti codes & 1.6 & 0.8 & 2.1 & 0.85 & 10.8 \\
\hline Spatial multiplexing in MIMO & 1.8 & 0.9 & 2.8 & 0.94 & 21.0 \\
\hline $\begin{array}{l}\text { Beamforming with multiple } \\
\text { antennas }\end{array}$ & 1.9 & 1.0 & 3.2 & 1.06 & 25.3 \\
\hline Small scale vs. large scale fading & 2.4 & 1.3 & 3.7 & 1.16 & 26.5 \\
\hline Shadow fading & 2.1 & 1.3 & 3.3 & 1.09 & 23.5 \\
\hline Path loss & 2.3 & 1.3 & 3.9 & 1.05 & 31.8 \\
\hline Coherence time of a wireless channel & 2.0 & 1.2 & 3.9 & 0.75 & 38.0 \\
\hline $\begin{array}{l}\text { Coherence bandwidth of a wireless } \\
\text { channel }\end{array}$ & 2.0 & 1.2 & 3.7 & 0.65 & 34.0 \\
\hline Wireless link budget analysis & 1.9 & 1.1 & 2.8 & 0.99 & 17.3 \\
\hline Frequency reuse in cellular systems & 2.4 & 1.4 & 3.6 & 1.34 & 23.3 \\
\hline Signal to interference ratio & 2.8 & 0.9 & 3.9 & 1.22 & 21.8 \\
\hline Handover in wireless networks & 2.5 & 1.2 & 2.6 & 1.29 & 2.0 \\
\hline Wireless localization & 1.9 & 0.9 & 3.4 & 0.88 & 29.3 \\
\hline $\begin{array}{l}\text { Why we need digital } \\
\text { communications over analog? }\end{array}$ & 3.1 & 1.2 & 4.8 & 1.33 & 34.8 \\
\hline Nyquist theorem & 3.0 & 1.0 & 4.4 & 1.12 & 28.0 \\
\hline Signal quantization & 2.7 & 0.9 & 4 & 1.01 & 26.3 \\
\hline Source coding & 2.5 & 1.2 & 4.2 & 1.20 & 34.0 \\
\hline Channel coding & 2.4 & 1.2 & 4.1 & 1.19 & 34.5 \\
\hline Downsampling/upsampling a signal & 2.4 & 1.1 & 4 & 1.20 & 32.5 \\
\hline Least square parameter estimation & 1.9 & 1.1 & 3.9 & 0.66 & 39.3 \\
\hline
\end{tabular}


main theme of Lab-3, and $43 \%$ improvement was observed in survey results for synchronization. Similarly, channel estimation and equalization are the main focus of Lab-4, which boasted $43.8 \%$ (wireless channel estimation) and $33.8 \%$ (wireless equalization) gain in survey results, respectively.

While the original lab package includes three additional experiments with OFDM (Labs 6-8), they were not included in the course offering due to time/hardware limitations. On the other hand, OFDM modulation is still studied extensively during the lectures. Results in Table 2 show that gain in OFDM modulation is only $28.5 \%$, which is considerably lower than other technical concepts such as synchronization and channel estimation, and may have been improved via a hands-on lab experiments. The concept of MIMO is covered only briefly in the lectures, and there is no lab experiment associated with this concept. This is also reflected in the low improvement (17\%) in students' learning perceptions on the topic of MIMO systems.

As a last remark, we would like to comment on the $39.3 \%$ gain observed in least square parameter estimation. Normally, least square estimation is a mathematical concept that is relatively challenging to understand. This concept has been introduced in the class, and examples are provided in the context of synchronization, equalization, and channel estimation.

Subsequently, students test this technique in lab experiments. Thus, even mathematically abstract concepts that are not directly related to wireless communications can be better learned using hands-on lab experiments. Coupling hands-on activities with lectures encourages students to make connections that may not occur otherwise.

\section{Feedback from the Students}

In the surveys, students have also provided extensive feedback about the different aspects of the course. In a question asking whether students have any suggestion for improving the lab to have a better learning experience, some of the answers from the students include the following:

- "The lab instructions were very detailed until the end. However, the part where we had to graph the I and Q components of the signal was not clear. Lab procedures could be improved."

- "Manual is too long. For pre-lab part, it does not clearly state what is expected from us, which makes us struggle much more than required to learn."

- "Better introduction to LabVIEW and the USRP (is needed)".

- "Since LabVIEW is new to many of us, it would be greatly helpful if the lab process were taken step-by-step. Perhaps we can start with an easy example, such as an AM Demodulator and understand what is happening in the process in LabVIEW."

- "Since we did not build the whole standing code in LabVIEW, sometimes it is difficult to even know what is to be done. A short instructional lecture before the lab would help."

- "Because of labs and HWs, workload is a bit more consuming most of the time in a week. This makes it difficult to spend time on other courses and research. Hoping the workload will be reduced."

- "An introduction of pre-lab is very helpful."

- "Have a lab session strictly for the pre-lab. It is harder than the actual lab." 
- "Only suggestion is to make the course a bit easy, as some other courses in the department has already been finished. And along with labs and project it becomes difficult to cope up with things."

- "Pre-Lab took was difficult and took some time. Most likely because of unfamiliarity with LabView and little knowledge of the default virtual instruments (VIs) that are available."

- "Lab work is not balanced. Giving all the work to pre-labs, leaving nothing to the actual lab is not feasible. This also causes loss of discipline in labs (since most of the work is on pre-labs, before the lab)."

These remarks from the students show that the workload of the course, which includes lectures, biweekly labs, course project, homework, and exams, is heavy. Note that the course does not even include all the eight labs that are available in the original teaching bundle, and skips labs 6-8 pertaining to OFDM systems to make the labs more manageable. Still, some students could not manage to carry out all assignments, due to their other workload in other courses. This is understandable, considering that committing to additional biweekly lab experiments in addition to regular weekly lectures takes extensive time. Further consideration should be given on how to reduce workload of the course, while still providing the technical content and hands-on experimentation in wireless communications. Another important issue is to tailor the course based on the students' backgrounds and levels at different universities.

Many of the students bring the issue of lengthy pre-labs, which students are expected to do before coming to the lab for conducting hands-on experiments. The lab experiments also require good LabVIEW proficiency, which may be challenging for students who are using LabVIEW Communications for the first time. One solution can be to merge pre-labs into longer lab sessions, which on the other hand require longer commitments from students and lab teaching assistants. One or two weeks of LabVIEW and USRP tutorials during the first 1-2 weeks of the course may also help students which are not competent in LabVIEW. One week of basic introduction has already been provided before starting the original labs, but this looks to be insufficient.

In response to another question on how useful the USRP lab experiments have been, some of the student responses include the following:

- "USRP labs gave a very good idea about the practical aspects of digital system designs."

- "It is a cool lab and helped a lot to understand the concepts for the class. We need more guidelines though."

- "The labs require a lot of time and effort, the simulator had a lot of errors, but once the factors were overcomed, the labs helped improve the foundation learned in the class."

- "I felt the labs had a very sharp learning curve. Pre-lab took very long time. Labs need to be more structured."

From these responses it can be understood that the students value the experience obtained from hands-on lab sessions. On the other hand, students who do not have sufficient background may really struggle with lab experiments. At the undergraduate level, students may take the course with limited knowledge of LabVIEW, Digital Signal Processing and Communication 
Theory. For universities which do not offer the necessary prerequisite courses to prepare students to this course, a simpler USRP course can be taught at the undergraduate level. In particular, NI has a separate and more introductory teaching bundle for undergraduate students, prepared by Rose-Hulman Institute of Technology ${ }^{13}$, for which students do not need extensive preliminary knowledge. It can serve as a good alternative for universities where a more advanced communication course cannot be taken at undergraduate level due to limited offering of communications courses.

Finally, in response to a question asking for any other suggestions for improving the learning experience from the class, following comments have been made:

- "The lab experiments were under-emphasized, labs were more difficult than the actual class."

- "More in class examples would help a lot. The course project is a good experience but working in groups would be better learning process."

- "Following equations from slides is hard. Slides are not efficient to study (explanations are not enough), book is not easy to follow. When I missed something in the class I could not know how to learn it (from which source)."

- "Explaining practical aspects of these concepts will be helpful."

- "TA should be patient to explain ideas in pre-labs and labs. I need some math background on estimation theory before selecting this course. Workload is a little bit heavy because of the pre-labs."

- "I don't think it is necessary to include homeworks in this course. There is already too much material."

These responses again emphasize the high workload for the course. Extensive amount of theory is introduced during the lectures. While hands-on experiments help in understanding some concepts, responses show that more in class examples are still needed. Course projects are, also, very appreciated by the students, since they can work on their own pace, on an application area which they are genuinely interested. The importance of a highly motivated and experienced teaching assistant is one of the key factors that impact the learning experience of the students.

\section{Concluding Remarks}

In this paper, we presented our experiences and findings from teaching a hands-on course on wireless communication to undergraduate and graduate students. The survey data show that hands-on experimentation is much appreciated by the students for learning wireless communication concepts. Technical concepts which have a hands-on learning component are perceived to be better learned by the students, when compared to technical concepts which are not directly tied to a lab experiment. The course is considered to have a heavy workload, and further studies are needed to understand whether the workload can be reduced while still delivering key technical skills to the students. Overall, the use of hands-on lab experiments in wireless communication courses is highly recommended. 


\section{References}

1. Mitola, J. (1995). The software radio architecture. IEEE Commun. Mag., 33(5), 26-38.

2. Mitola, J. (2000). Cognitive radio: an integrated agent architecture for software-defined radio. Ph.D. dissertation, Royal Institute of Technology (KTH), Stockholm, Sweden.

3. Robert W. Heath. EE 471C / EE 381V: Wireless Communications Lab. University of Texas Austin. Course Website. http://www.profheath.org/teaching/ee-371c-ee-381v-wireless-communications-lab/

4. National Instruments. "Digital Communications Teaching Bundle". Teaching Bundle Website. http://sine.ni.com/nips/cds/view/p/lang/en/nid/213007

5. Shankar, P. M. \& Eisenstein, B. A. (2000). Project-based instruction in wireless communications at the junior level. IEEE Transactions on Education, 3, 245-249.

6. Padgett, W. T., Black, B. A., \& Ferguson, B. A. (2006). Low-frequency wireless communications system Infrared laboratory experiments. IEEE Transactions on Education, 49(1), 49-57.

7. Sarkar, N. I., \& Craig, T. M. (2006). Teaching wireless communication and network fundamentals using wi-fi projects. IEEE Transactions on Education, 29(1), 98-104.

8. Sanguino, T. d., Lopez, C. S., \& Hernandez, F. A. (2013). WiFiSiM: An educational tool for the study and design of wireless networks. IEEE Transactions on Education, 56(2), 149-155.

9. Frolik, J., \& Weller, T. M. (2002). Wireless sensor systems: An approach for a multiuniversity course design. IEEE Transactions on Education, 45(2), 135-141.

10. Taslidere, E., Cohen, F. S., \& Reisman, F. K. (2011). Wireless sensor networks - A hands-on modular experiments platform for enhanced pedagogical learning. IEEE Transactions on Education, 54(1), 24-33.

11. Dawy, Z., Husseini, A., Yaacoub, E., \& Al-Kanj, L. (2010). A wireless communications laboratory on cell network planning. IEEE Transactions on Education, 53(4), 653-661.

12. Guzelgoz, S., \& Arslan, H. (2010). A wireless communications systems laboratory course. IEEE Transactions on Education, 53(4), 532-541.

13. National Instruments. Introduction to Communications Teaching Bundle With $2 X 2$ MIMO. http://sine.ni.com/nips/cds/view/p/lang/en/nid/213146.

14. National Instruments. SDR for Education. http://www.ni.com/sdr/education/. 